\title{
Neurobiological mechanisms associated with facial affect recognition deficits after traumatic brain injury
}

\author{
Dawn Neumann, PhD \\ Indiana University School of Medicine \\ Department of Physical Medicine and Rehabilitation \\ Rehabilitation Hospital of Indiana \\ 4141 Shore Drive \\ Indianapolis, IN 46254 \\ Email: dmneuman@iupui.edu \\ Phone: 317-329-2188 \\ Brenna C. McDonald, PsyD, MBA \\ Indiana University School of Medicine \\ Department of Radiology and Imaging Sciences \\ Indiana University Center for Neuroimaging \\ 355 W. $16^{\text {th }}$ St., GH Suite 4100 \\ Indianapolis, IN 46202 \\ Email: mcdonalb@iupui.edu \\ John West, MS \\ Indiana University School of Medicine \\ Department of Radiology and Imaging Sciences \\ Indiana University Center for Neuroimaging \\ 355 W. $16^{\text {th }}$ St., GH Suite 4100 \\ Indianapolis, IN 46202 \\ Email: jdwest@iupui.edu \\ Michelle A. Keiski, PhD \\ Indiana University School of Medicine \\ Department of Physical Medicine \& Rehabilitation \\ Indiana University Center for Neuroimaging \\ 355 W. $16^{\text {th }}$ St., GH Suite 4100 \\ Indianapolis, IN 46202 \\ Email:michellekeiski@gmail.com \\ Yang Wang, MD \\ Department of Radiology \\ Department of Biophysics \\ Medical College of Wisconsin \\ 8701 Watertown Plank Road \\ Milwaukee, WI 53226 \\ Email: yangwang@mcw.edu
}

Key words: Neuroimaging, emotion, facial affect recognition, traumatic brain injury, functional MRI, fMRI

This is the author's manuscript of the article published in final edited form as:

Neumann, D., McDonald, B. C., West, J., Keiski, M. A., \& Wang, Y. (2015). Neurobiological mechanisms associated with facial affect recognition deficits after traumatic brain injury. Brain Imaging and Behavior, 1-12.

http://doi.org/10.1007/s11682-015-9415-3 


\begin{abstract}
The neurobiological mechanisms that underlie facial affect recognition deficits after traumatic brain injury (TBI) have not yet been identified. Using functional magnetic resonance imaging (fMRI), study aims were to 1) determine if there are differences in brain activation during facial affect processing in people with TBI who have facial affect recognition impairments (TBI-I) relative to people with TBI and healthy controls who do not have facial affect recognition impairments (TBI-N and HC, respectively); and 2) identify relationships between neural activity and facial affect recognition performance. A facial affect recognition screening task performed outside the scanner was used to determine group classification; TBI patients who performed greater than one standard deviation below normal performance scores were classified as TBI-I, while TBI patients with normal scores were classified as TBI-N. An fMRI facial recognition paradigm was then performed within the 3T environment. Results from thirty-five participants are reported (TBI-I=11, TBI-N=12, and $\mathrm{HC}=12$ ). For the fMRI task, TBI-I and TBI-N groups scored significantly lower than the HC group. Blood oxygenation level-dependent (BOLD) signals for facial affect recognition compared to a baseline condition of viewing a scrambled face, revealed lower neural activation in the right fusiform gyrus (FG) in the TBI-I group than the HC group. Right fusiform gyrus activity correlated with accuracy on the facial affect recognition tasks (both within and outside the scanner). Decreased FG activity suggests facial affect recognition deficits after TBI may be the result of impaired holistic face processing. Future directions and clinical implications are discussed.
\end{abstract}




\section{BACKGROUND}

The ability to perceive and interpret emotions displayed by others is critical to successful interpersonal interactions. It is a key factor in shaping optimal reactions and behaviors towards others (e.g., consoling a sad friend). Emotions are predominantly expressed through nonverbal cues, such as facial expressions, tone of voice, and body language (Nowicki and Mitchell 1998). Hence, an individual may rely on one or more of these nonverbal cues in deducing the emotional state of others to guide social interactions (Fusar-Poli et al. 2009). The ability to recognize emotions from facial expressions is known to be impaired in several neurological disorders, including autism, schizophrenia, and traumatic brain injury (TBI) (Babbage et al. 2011; Edwards et al. 2002; Neumann et al. 2015; Ogai et al. 2003). The current study focuses on facial affect recognition impairments after moderate to severe TBI.

According to a meta-analysis published in 2011, 39\% of people with moderate to severe TBI had significant facial affect recognition deficits, compared to only $7 \%$ of healthy controls (Babbage et al. 2011). With 373,000 Americans estimated to suffer a non-fatal moderate to severe TBI each year (Centers for Disease Control and Prevention), it can be projected that over140,000 individuals are likely to sustain a TBI associated with facial affect recognition impairments annually in the US alone. The fact that research has tied these deficits to emotion dysregulation, behavioral problems, and inappropriate social interactions (Knox and Douglas 2009; Spikman et al. 2013), underscores the importance of learning more about the factors that contribute to facial affect recognition impairments after TBI. Despite the prevalence and negative implications of facial affect recognition deficits, no research to date has investigated the neurobiological mechanisms underlying these impairments in the TBI population. Efforts to elucidate these mechanisms may enhance affect recognition treatment approaches for people with TBI.

Neuroimaging studies in healthy controls show that facial affect recognition engages a widely distributed neural network, including structures within limbic and subcortical regions, 
prefrontal cortex, temporal and parietal lobes, and occipital cortex (Fusar-Poli et al. 2009;

Sabatinelli et al. 2011). These brain regions have been associated with functions that contribute to successful facial affect recognition. Many researchers and theorists postulate that there are three main elements that contribute to facial affect recognition: perception, emotion replication and experience, and conceptual understanding (Neumann et al. 2014a). Neuroimaging studies on healthy controls and clinical populations, as well as behavioral studies in participants with focal brain lesions, have helped link the elements of facial affect recognition with specific neuroanatomical regions and networks. Briefly, the elements of facial affect recognition and associated brain structures are described below.

Perception. Studies indicate that healthy controls perceive features of a face as a single gestalt representation; in other words, faces are processed holistically, as an integral unit. (Maurer et al. 2002). Holistic processing has characteristically been associated with activation in the fusiform gyrus (Kanwisher and Yovel 2006). Because the fusiform gyrus appears to have stronger responses to emotional faces than neutral faces (especially in the right hemisphere), it has been suggested that the fusiform gyrus is also implicated in the processing of facial affect, rather than a role restricted to perceptual processing of faces without emotional cues (Ganel et al. 2005; Kanwisher and Yovel 2006).

In addition to processing faces as a gestalt, there is also evidence from research using eye-tracking technology that suggests healthy controls spend more time looking at the eye region than other areas of the face (e.g. the mouth) when viewing affective faces. (Adolphs 2002; Vassallo et al. 2009). In contrast, participants with autism, who characteristically have facial affect recognition impairments, typically spend more time looking at the mouth (Klin et al. 2002; Pelphrey et al. 2002). Neuroimaging studies have shown that attention to the eye region is typically associated with greater activation in the amygdala (Dalton et al. 2005), and that damage to the amygdala results in reduced fixations on the eyes (Adolphs et al. 2005). 
Emotion replication and experience. Emotion replication and experience is when one reproduces and/or feels others’ emotions, including facial expressions and physiological responses, within oneself (Adolphs 2002; Phillips et al. 2003). The amygdala, insula, somatosensory-related cortices (including supramarginal and angular gyri), basal ganglia, cingulate, and ventromedial prefrontal cortices have been identified as important regions for emotion replication and experience; these structures are commonly activated in healthy controls during neuroimaging studies evaluating mechanisms associated with facial affect recognition. (Adolphs 2002; Phillips et al. 2003; Neumann et al. 2014a).

Conceptual understanding. A conceptual understanding of a specific emotion reflects an aggregation of past memories, physical and emotional experiences, and the situational context surrounding that emotion. The medial prefrontal cortex and inferior frontal gyrus are areas that have been associated with conceptual understanding of emotions (Adolphs 2002; Neumann et al. 2014a). Furthermore, research findings show that these regions are frequently activated in healthy controls during neuroimaging studies that involve labeling facial affect (Fusar-Poli et al. 2009; Sabatinelli et al. 2011; Neumann et al. 2014a).

In summary, past research has linked specific neuroanatomical structures with functions responsible for the different elements of facial affect recognition. Importantly, this groundwork enables functional and meaningful interpretations to be made from neuroimaging studies that aim to understand facial affect recognition deficits in populations with impairments to these areas. For instance, numerous neuroimaging studies have already investigated the neurobiological mechanisms underlying facial affect recognition impairments in people with autism and schizophrenia (Harms et al. 2010; Li et al. 2010). The most consistent finding across these studies has been reduced activation in the amygdala and fusiform gyrus during facial affect recognition tasks relative to controls (Harms et al. 2010; Li et al. 2010). This suggests that deficient affect recognition in people with autism and schizophrenia may be the result of problems with perceptual processing of facial expressions and/or emotional experience and replication. The 
notion that abnormal perceptual processing is contributing to affect recognition deficits in autism is further supported by the deviant visual scanpaths demonstrated by participants with autism in eye tracking studies described earlier (Dalton et al. 2005; Klin et al. 2002).

To date, no studies have used functional magnetic resonance imaging (fMRI) to examine the neurobiological responses during an emotional face processing task in people who have had a moderate to severe TBI. Thus, the overall objective of the current study was to use fMRI to examine candidate mechanisms that underlie impaired facial affect recognition after TBI. These preliminary findings will begin to shed light on which element(s) of the facial affect recognition process are impaired after TBI: perception, emotion replication and experience, and/or conceptual understanding. Our first aim was to determine if there are differences in brain activation during facial affect processing in people with TBI who have facial affect recognition deficits (TBI-I) to people with TBI and healthy controls who do not have facial affect recognition deficits (TBI-N and HC, respectively). Our second aim was to identify relationships between fMRI activity and behavioral scores on facial affect recognition tasks. Since people with TBI perform similarly on behavioral tasks of emotion recognition to people with autism and schizophrenia, we hypothesized that people with TBI would also have decreased activations in the fusiform gyrus and the amygdala.

\section{METHODS}

\section{Study Population}

We recruited three groups of participants: TBI-I (TBI with impaired facial affect recognition), TBI-N (TBI with normal facial affect recognition), and HC (healthy control with normal facial affect recognition). Participants with TBI were a convenience sample recruited from a local brain injury rehabilitation facility, brain injury support groups, and referrals from other research studies. Age and gender-matched healthy controls were a convenience sample recruited from local research study advertisements. Our target was to recruit 12 participants per 
group, consistent with sample sizes in neuroimaging studies on affect processing in people with autism (Piggot et al. 2004; Wang et al. 2004).

General participation criteria (all participants). Based on a priori inclusion criteria, all participants were: right-handed, 18-65 years of age, not currently pregnant or lactating, and deemed safe for MRI scanning in the 3T environment. All participants were administered line cancellation, facial matching, and facial affect recognition tasks during an initial screening visit. Participants were excluded from participating in the fMRI visit if they demonstrated visual neglect, gross visual impairment, significant visual-perceptual deficits affecting general face processing, prominent aphasia, gross auditory impairment, or substantially impaired motor control with their right hand during the screening visit, since these deficits could interfere with their ability to meaningfully complete the fMRI task or otherwise introduce confounds in interpreting the imaging results.

Participation criteria for participants with TBI. All participants had sustained a moderate to severe closed TBI, which required they met one of the following severity criteria: Glasgow Coma Score $\leq 12$, or post-traumatic amnesia (PTA) $\geq 24$ hours, or loss of consciousness (LOC) $\geq 30$ minutes, based on medical record review or patient/family report. All participants were at least three months post-injury at the time of evaluation. TBI participants were excluded if their injury was predominantly penetrating in nature (e.g., gunshot wound) or if they had a premorbid history of neurological (e.g., epilepsy) or psychiatric disorder (e.g., schizophrenia).

On the initial visit, participants completed a standardized test of facial affect recognition (Diagnostic Assessment of Nonverbal Accuracy-2 Adult Faces, DANVA2-AF) to determine group classification: TBI-I if performance was more than one standard deviation below the mean per DANVA2-AF age-corrected normative data; or TBI-N if performance was above this level.

Participation Criteria for Healthy Controls. In addition to the general participation criteria stated above, all healthy controls were free from a past or current history of neurological 
or psychiatric disorder. Healthy controls were also required to score within the normal range on the DANVA2-AF.

Forty-five participants were screened for the study (TBI=32; HC=13). Of the 32 screened participants with TBI, 12 (37.5\%) were impaired at facial affect recognition. Of the participants with TBI, 24 qualified (TBI-I=12; TBI-N=12). One participant did not qualify because he scored in the impaired range on the face matching task, indicating some general face processing impairments beyond facial expressions; and seven were not eligible for further participation because they scored within normal range on the DANVA-2AF and the TBI-N group was full. Of the HC group, 12 qualified and one was excluded because he performed outside the normal range on the DANVA2-AF. A total of 36 subjects, 12 per group, participated in the full study that included fMRI. However, because one participant in the TBI-I group could not stay awake in the scanner and did not complete the scanner tasks, this participant was excluded from all analyses, resulting in sample sizes of TBI-I=11; TBI-N=12, and $\mathrm{HC}=12$. Our final sample $(n=35)$ was $54.3 \%$ male, an average of 39.69 years old and had an average of 15.37 years of education. Eighty percent of participants were Caucasian, 14.3\% black, 2.9\% Hispanic, and 2.9\% 'other'. On average, participants with TBI were 10.77 years post-injury (S.D.=9.20; range: $0.6-36$ ), had a loss of consciousness for 17.79 days (S.D.=23.11; range: 0-90; $n=19$ ), and had posttraumatic amnesia for 23.27 days (S.D.=16.16; range: 2-60; $n=15$ ). GCS was only available for four participants and is, therefore, not reported. Seventy-four percent of participants were injured in a motor vehicle accident. See Table 1.

\section{Measures}

Main outcome measures were two facial affect recognition tasks described below (DANVA2-AF screening measure; neuroimaging task [see fMRI Facial Affect Recognition Paradigm]) and brain activation during the neuroimaging task. Measures of depression and anxiety were also administered to assess participant affect and examine potential relationships with task performance. 
Diagnostic Assessment of Nonverbal Accuracy-2 Adult Faces (DANVA2-AF) (Nowicki and Duke 1994). The DANVA2-AF is a standardized measure of facial affect recognition with age-corrected norms. Twenty-four faces portraying specific emotions were presented one at a time on a computer screen for five seconds. Participants were asked to choose from a list of emotions which best described the facial expression: happy, sad, angry, or fearful. Responses after five seconds were counted as incorrect. This measure was chosen over other facial affect recognition measures that include additional emotions (e.g. surprise and disgust) for several reasons. First, this test has been used in numerous studies to evaluate affect recognition performance in the TBI population (Neumann et al. 2012; Neumann et al. 2014b; Spell and Frank 2000; Neumann et al. 2015). Studies show that it correlates well with other facial affect recognition measures and has good test-retest reliability (McIntire et al. 1997; Spell and Frank 2000). Additionally, the DANVA2-AF was chosen because it evaluates the same four emotions (different face stimuli) as the fMRI facial affect recognition task [described below].

Patient Health Questionnaire-9 (PHQ-9)(Kroenke et al. 2001). The PHQ-9 is a nineitem, self-administered depression questionnaire. The nine statements describe symptoms that are consistent with the DSM-IV diagnostic criteria for depressive disorders. Participants must indicate the frequency of symptom occurrence using a 0 to 3 scale, from "not at all” to "nearly every day”. The PHQ-9 has been demonstrated to be a reliable and valid tool for measuring depression in the general population as well as in patients with TBI (Fann et al. 2005).

State Trait Anxiety Inventory (STAI) (Spielberger et al. 1970). The State Trait Anxiety Inventory (STAI) is a self-report measure of state and trait anxiety (20 items each). Higher scores indicate more trait or state anxiety. The STAI has been shown to have concurrent validity with the Taylor Manifest Anxiety Scale, the IPAT Anxiety Scale, and the Multiple Affect Adjective Check List (Spielberger et al. 1995) and has been used in previous studies to assess anxiety in people with TBI. (Curran et al. 2000; Marsh 1998)

\section{fMRI Facial Affect Recognition Paradigm}


The neuroimaging face emotion identification task (Gur et al. 2002a; Gur et al. 2002b) is comprised of four conditions, one for each target expression: happy, sad, anger, or fear. These conditions were each presented as a separate task and in a counterbalanced order. Each condition included four blocks of emotion identification, alternating with five blocks where a single scrambled face with a central cross-hair for fixation was presented (See Figure 1). Each emotion identification block contained 30 faces, which included target faces for each of the different emotion conditions (e.g., fear), foil faces displaying the nontarget conditions (e.g., happy, sad, angry), and neutral faces. Over the four blocks within each task run the 120 faces presented included 32 target, 32 foil, and 56 neutral faces in a pseudorandom sequence. Faces were on the screen for 2.25 seconds, and participants were instructed to identify “Target” (e.g., “Anger”) or “Other/Neutral” from the left and right buttons of a response pad, respectively. Response cues remained visible throughout the face blocks and a box appeared to indicate the subject's response (once made) without providing feedback regarding accuracy. The same stimuli were used throughout the four tasks serving as targets or foils, depending on the condition, and stimuli were equally distributed for sex and balanced for ethnicity. Each task run was 6:31 in duration, for a total task duration of 26:04. Participants completed a brief training on this paradigm before entering the scanner to ensure they understood how to perform the task.

-INSERT FIGURE 1

\section{Study Procedures}

All participants provided informed consent prior to any testing. This study involved an initial screening visit, which was followed by an imaging visit for participants who were eligible for the fMRI portion of the study. During the screening visit, participants were administered a demographic and medical history questionnaire to screen for inclusion and exclusion criteria. Participants were administered a line cancellation task to exclude any potential participants with visual neglect, and a neutral face matching task from the Florida Affect Battery was used to exclude participants with gross visual-perceptual impairments hindering perception of facial 
characteristics. The DANVA-2 AF was also administered during the initial visit in order to measure facial affect recognition and determine group membership, as described above. Lastly, the STAI and the PHQ-9 were administered to examine participant affect. Participants were called at a later date to notify them of eligibility and to set up a second visit for the fMRI portion of the study if they qualified.

\section{Image Acquisition}

All scans were acquired on the same 3T Siemens Skyra scanner using a 32-channel head coil. A gradient-echo, echo-planar sequence provided whole brain coverage for fMRI:

$\mathrm{TR}=2250 \mathrm{~ms}, \mathrm{TE}=29 \mathrm{~ms}, \mathrm{FOV}=24 \mathrm{~cm}, 39$ interleaved $3.5 \mathrm{~mm}$ thick contiguous axial slices, yielding a 80x80 matrix with $3 \mathrm{~mm}^{2}$ in-plane resolution. In addition to the fMRI paradigm, all participants underwent structural MRI scans, including MPRAGE, FLAIR, and SWI sequences in order to characterize the nature and extent of their injuries for TBI participants and to exclude significant unreported abnormalities in healthy controls. All scans were formally reviewed and interpreted by a clinical neuroradiologist.

\section{DATA ANALYSIS}

Behavioral Data Analysis. Descriptive statistics, including means, standard deviations, and percentages were calculated for participant characteristics and task performance. A Chisquare test was used to compare gender differences across groups. Independent t-tests were used to compare differences in injury characteristics between the two TBI groups (alpha<0.05). When statistically appropriate, differences across the three groups (TBI-I, TBI-N, and HC) were calculated with one-way ANOVAs, and when significant, were followed by Bonferroni-corrected pair-wise comparisons (alpha $=0.02$ for 3 comparisons). However, when homogeneity of variance tests indicated significantly different variances, the Kruskal-Wallis test was used instead; in cases of significant findings $(\mathrm{p}<0.05)$, pair-wise comparisons were performed with the Wilcoxon Rank Sum test, applying a Bonferroni correction (alpha=0.02 for 3 comparisons). Spearman's Rho was 
used to examine correlations between facial affect recognition performance, reaction time and brain activity (alpha<0.05).

Image Analysis. Imaging data preprocessing and analyses were conducted in SPM8. In brief, after correction of slice timing, spatial realignment was performed on functional images to remove minor motion-related signal change. fMRI time series were then co-registered to the participant’s MPRAGE volume, and normalization parameters from segmentation of the MPRAGE were used for normalization into Montreal Neurological Institute atlas space. All volumes were resampled to $2 \mathrm{~mm}^{3}$ isotropic voxels and smoothed to a FWHM of $6 \mathrm{~mm}$. Realignment generated six motion parameters which were entered as covariates at the individual subject level. Contrast images comparing blocks of viewing faces to blocks of viewing the scrambled face were created for each task for each participant. These contrast images were then used in second-level multi-subject (between-group) voxel-wise analyses. Voxel-wise random effects analyses for fMRI were conducted using a full factorial model as implemented in SPM8 to construct maps of voxels in which activation differed as a function of group across all four task conditions combined. Voxel-level (peak) significance values represent the chance under the null hypothesis of finding a voxel with as great as or greater a height threshold (Z). Cluster-level significance can be interpreted as the probability under the null hypothesis of finding a cluster with as great as or greater a number of voxels. Findings are reported at an overall $\mathrm{p}_{\text {crit }}=0.001$; only clusters with cluster-level $\mathrm{p}_{\text {uncorr }}<0.05$ were considered to be significant. Activation values for the significant cluster from between-group comparisons were extracted using MarsBaR for further statistical analyses.

\section{RESULTS}

\section{Comparisons of Sample Characteristics.}

See Table 1 for descriptive statistics and group comparisons. The three groups did not significantly differ in age, sex, or years of education. Additionally, they did not significantly 
differ with respect to symptoms of depression or anxiety. Comparisons between the two TBI groups revealed they were similar with respect to injury severity and chronicity.

Regarding the structural MRI scans, the majority of TBI participants demonstrated features compatible with remote TBI, including the presence of focal encephalomalacia, mild to moderate generalized cerebral atrophy and ventricular prominence, and scattered abnormalities on FLAIR and SWI representing white matter injury and hemisoderin deposition, respectively. For the healthy controls, the structural scans were typically unremarkable, with the exception of a few participants for whom minor abnormalities were noted on FLAIR, likely compatible with small vessel ischemia, often age-appropriate, in addition to minor incidental findings (e.g., small nonneoplastic cyst).

Of note, several of the participants were taking prescription medications that could affect their mood, cognition, or neural activation. For example, several participants were taking SSRIs, SNRIs, and NDRIs for depression or anxiety. Other common medications included: methylphenidate or donepezil for cognitive enhancement; opiates or anticonvulsants (e.g., gabapentin) for pain; anticonvulsants (e.g., divalproex, oxcarbazepine, lamotrigine) for posttraumatic seizure disorders or mood stabilization; trazodone for sleep; and (more rarely) benzodiazepines such as diazepam. These medications were primarily prescribed to the TBI participants; there was one healthy control who was taking antidepressants for mild depression. INSERT TABLE 1

\section{Facial Affect Recognition Behavioral Performance Group Differences}

With respect to accuracy scores on the DANVA2-AF screening test and the neuroimaging facial affect recognition task, the Levine test of homogeneity of variance indicated significant group differences in variances ( $p=0.027$ and $p=0.014$, respectively). Therefore, the Kruskal-Wallis test was performed to compare differences on task scores.

Z-scores were used to calculate group differences on the DANVA2-AF; by design, there was a significant group difference $\left(\chi^{2}=22.17, \mathrm{p}<0.001\right)$. Pairwise comparisons with the Wilcoxon 
Rank Sum test revealed that the scores for the TBI-I group were significantly lower than the TBI$\mathrm{N}$ group $(\mathrm{z}=-4.067, \mathrm{p}<0.001)$ and the HC group $(\mathrm{z}=-4.070, \mathrm{p}<0.001)$ on the DANVA2-AF, as would be expected based on group inclusion criteria. There was no significant difference between the TBI-N group and the $\mathrm{HC}$ group $(\mathrm{z}=-0.435, \mathrm{p}=0.663)$. On the neuroimaging facial affect recognition task, a significant difference was also found between groups $\left(\chi^{2}=11.50, \mathrm{p}=0.003\right)$. Pairwise comparisons indicated that the TBI-I and TBI-N groups had significantly lower accuracy than the HC group ( $\mathrm{z}=-2.96, \mathrm{p}=0.003 ; \mathrm{z}=-2.55, \mathrm{p}=0.011$, respectively). No significant differences were identified between the two TBI groups $(\mathrm{z}=-1.45$, $\mathrm{p}=0.147)$.

For the neuroimaging task, we also compared reaction time differences across the groups. The Levine's test of homogeneity of variances indicated that the reaction time variances were not significantly different across groups ( $\mathrm{p}>0.05$ ); thus, a one-way ANOVA was used for analyses. Results indicated that reaction times significantly differed across groups ( $F=5.78, \mathrm{p}=0.007)$. Posthoc pairwise comparisons using a Bonferroni correction revealed that the TBI-I group had significantly longer reaction times than the HC group ( $\mathrm{p}=0.007)$; no other group comparisons were significant. A correlational analysis that included all three groups revealed a significant association between reaction time and the neuroimaging task performance scores $(\mathrm{r}=-0.526$, $\mathrm{p}=0.001$ ). Descriptive statistics are provided in Table 2.

---INSERT TABLE 2

\section{fMRI Results}

Task Main Effects. Images depicting the main effect for the fMRI facial affect recognition task for all three participant groups combined are presented in Figure 2. This depicts the broader network elicited by the task, the pattern of which is similar for all three of the groups and is compatible with prior research literature on facial affect recognition. Specifically, activation was observed in bilateral fusiform, inferior and superior frontal, and superior temporal gyri, bilateral amygdalae, basal ganglia, and midbrain, and right cerebellum (see Table 3 for activation values and neuroanatomical labels). 
Between-Group Comparisons. HC participants had significantly greater activation during facial affect recognition in the right fusiform gyrus compared to the TBI-I group (Figure 3). No other significant group differences were observed at the selected statistical threshold (see Table 3 for activation coordinates, cluster sizes, statistical significance levels, and neuroanatomical labels). When peak activation values from the fusiform gyrus cluster were examined, it was apparent that the TBI-N group showed intermediate activation between the HC and TBI-I groups in this region, even though group differences did not meet criteria for statistically significant differences at this stringent statistical threshold (Figure 3).

-INSERT TABLE 3 and FIGURES 2 \& 3

\section{Associations between Brain Activity and Facial Affect Recognition}

Spearman's correlational analysis, including all participant groups, was performed to determine the association between facial affect recognition and activation in the right fusiform gyrus. Activity in the right fusiform gyrus was significantly associated with participants’ facial affect recognition screening scores on the DANVA2-AF $(r=0.406, \mathrm{p}=0.015)$ and neuroimaging facial affect recognition task scores $(r=0.488, p=0.003)$. These findings indicate that better performance was associated with greater fusiform activity. Fusiform activation was not significantly associated with reaction time on the neuroimaging task, though a trend suggested that greater activation was associated with faster reaction time $(r=-0.303, \mathrm{p}=0.076)$.

\section{DISCUSSION}

To date, this is the first study to examine the neurobiological mechanisms associated with facial affect recognition deficits after moderate to severe TBI. Neuroimaging studies in healthy controls have shown that facial emotion perception engages a large distributed network of structures, including limbic and subcortical regions, prefrontal cortex, temporal and parietal lobes, and occipital cortex. We observed activation involving similar circuitry across all three of our participant groups. However, there was one main neural activation difference that distinguished the impaired TBI group from the HC group. Our TBI-I group had significantly less 
activation in the right fusiform gyrus compared to HCs during the facial affect recognition task, consistent with our a priori hypotheses and prior literature findings in autism and schizophrenia (Edwards et al. 2002; Li et al. 2010). Additionally, there was a trend suggesting that right fusiform gyrus activation for the TBI-N group was intermediate to that in the HC and TBI-I groups.

With respect to performance, both the TBI-I and TBI-N groups had significantly lower scores on the neuroimaging facial affect recognition task compared to healthy controls; also, TBII participants had significantly longer reaction times than healthy controls. Facial affect recognition performance was associated with fusiform gyrus activation, demonstrating that greater activation in the fusiform gyrus was associated with better facial affect recognition, on both the screening measure of facial affect recognition and the neuroimaging facial affect recognition tasks. There was a trend toward greater activation being associated with faster fMRI reaction speed.

Understanding the behavioral mechanisms associated with the fusiform gyrus is particularly important for advancing our knowledge about the factors that are contributing to facial affect recognition deficits after TBI. The fusiform gyrus has typically been associated with face processing, particularly holistic face processing. Holistic processing is considered to be fundamental to face identity recognition and facial affect recognition (Avidan et al. 2011; Ganel et al. 2005; Maurer et al. 2002). Holistic face processing is assessed with tasks that manipulate one’s ability to perceive faces as a gestalt (e.g. Face Inversion Task (Maurer et al. 2002)). When task conditions disrupt the holistic percept, face recognition performance is typically altered in healthy controls (the "task effect”) (Rossion 2013; Rossion and Gauthier 2002). Neuroimaging studies in healthy controls reliably report greater activation in the fusiform gyrus during "intact" holistic face processing conditions, and conversely show decreased fusiform activation during conditions that disrupt the gestalt percept (Kanwisher and Yovel 2006). Additionally, studies have found that patients with focal lesions to the fusiform gyrus typically do not show the task 
effect, providing further evidence that the fusiform gyrus is critical to the gestalt processing of faces (Busigny et al. 2010; Busigny et al. 2014; Schiltz et al. 2010).

While the majority of studies described immediately above used neutral faces for stimuli, some studies have used emotional faces when evaluating holistic face processing (Ganel et al. 2005; Kanwisher and Yovel 2006). Notably, these studies have reported that fusiform gyrus activation (particularly right fusiform activation) is even more robust in response to emotional faces than to neutral faces (Ganel et al. 2005; Kanwisher and Yovel 2006). These findings have been used to posit a crucial role of the fusiform gyrus (and holistic facial processing) in detecting emotions from facial expressions, rather than a role restricted to facial identification (Ganel et al. 2005). It seems reasonable to hypothesize that integration of facial features (e.g., eyebrows, eyes, mouth) would provide a faster and more accurate representation of an expressed emotion than individual features, since the facial features typically work synergistically to convey emotions. However, other researchers do not believe that the fusiform gyrus is directly involved in processing facial affect. Instead, they believe that observed increases in fusiform activity merely represent a byproduct of increased activity in the amygdala, given the close proximity of these two structures (Dolan et al. 2001).

In contrast to our hypothesis, and findings from studies in autism and schizophrenia, our TBI-I impaired participants did not have reduced activation in the amygdala compared to the TBI-N and HC groups. Past studies have linked the amygdala with emotional replication and experience (Phillips et al. 2003), as well as time spent fixating on the eyes when processing emotional faces (Adolphs et al. 2005; Dalton et al. 2005). Thus, our results suggest that facial affect recognition impairments after TBI might not be related to problems with emotion replication or specifically attending to relevant facial features (e.g. the eyes). It should be noted, however, that our analytic approach of the neuroimaging task contrasted recognition of emotional and neutral faces versus a baseline condition of viewing a scrambled face. Since we did not specifically compare brain activation during processing of emotive faces versus neutral faces (due 
to limitations of the task), our findings may not only reflect mechanisms specific to processing facial affect per se, but may also be indicative of general face processing while explicitly attempting to identify affect. Because the amygdala is known to respond to emotion, direct comparison of activation during emotional versus neutral face processing might more directly detect group differences in this region. Consequently, future neuroimaging studies that plan to evaluate facial affect recognition impairments after TBI, should employ tasks that allow direct comparison of emotional versus neutral faces in order to determine brain activation differences specific to deficits in processing emotions from faces.

It is important to distinguish between face perception, face identity recognition, and facial affect recognition, particularly since prior studies have shown that these may engage dissociable networks, even though they share some interrelated structures (Narumoto et al. 2001; Winston et al. 2004). Of note, none of our participants exhibited the striking classical syndrome of prosopagnosia or gross visual-perceptual deficits hindering general facial perception, as indicated by their performance on the neutral face-matching task. Their deficits were more specific to emotion recognition per se. Furthermore, fusiform activity in the right hemisphere correlated with accuracy on behavioral measures of facial affect recognition. Taken together, our findings suggest that reduced activation in the right fusiform gyrus is related to facial affect recognition deficits in TBI per se, rather than other visual-perceptual aspects of facial perception (such as basic visual discrimination of features). Given that the fusiform gyrus has been routinely linked with holistic face processing (Rossion 2013; Kanwisher and Yovel 2006), we propose that the behavioral mechanism underlying facial affect recognition impairments after TBI may be related to deficits with integrating face features into a gestalt representation. This postulation should be empirically tested in future studies, ideally including both behavioral measures of holistic processing and neuroimaging with a larger sample size. If future studies identify problems with holistic face processing, and associate this deficit with facial affect recognition impairments after TBI, it would have important treatment implications. 
Although a few treatment regimens have been developed to help people with TBI improve emotion recognition from faces, holistic face processing was not directly targeted for improvement in any of these programs. This may be one reason why treatment with these programs have only shown small to moderate effect sizes in evidence-based studies. (Bornhofen and McDonald 2008; Neumann et al. 2015). It is certainly possible that treatment effect sizes would be larger if holistic facial processing was incorporated as a therapeutic target within the treatment programs. Should further studies confirm that holistic processing is a key component of facial affect recognition impairment after TBI, we would indeed recommend pursuing these changes to therapeutic regimens.

\section{Limitations}

A few limitations warrant brief discussion. Our small sample size may be considered a limitation; however, it is comparable to previous studies investigating the neurobiological underpinnings of emotion perception in other patient populations (e.g., autism) (Piggot et al. 2004; Wang et al. 2004). By matching for age and gender across our three groups, we were able control the potential influence of these extraneous variables on our outcomes. Furthermore, the strategic approach to include participants with TBI who did not have facial affect recognition impairments (TBI-N) helped to ensure activation differences in the TBI-I group were the result of emotion perception deficits in particular, and not just differences due to brain injury in general.

Although our groups appeared to be fairly homogeneous with respect to anxiety, the groups did appear to vary with respect to depressive symptoms. We suspect the group comparison on depression scores did not meet statistical significance due to the small sample size. The fact that our TBI patients had experienced challenges with depression is certainly supported by their medication regimens. Some of these confounding issues could have feasibly impacted the observed pattern of neural activation in response to the affect recognition paradigm. For example, the presence of depression has been demonstrated to influence recognition of sad and neutral faces, which may in turn affect neural responses (Bourke et al. 2010). Since our design prevents 
us from looking at responses to specific facial expressions, we cannot determine if mood symptoms had an impact on responses to emotional faces in this study.

Another potential issue is the heterogeneity of psychoactive medications taken by the TBI participants, including antidepressants, anxiolytics, anticonvulsants, dopamimetics, and (more rarely) benzodiazepines. Some of these medications could potentially alter the fMRI activation signals via a general effect on neuronal excitability or a more specific effect on mood. The potential impact of these medications as a confounding variable could not be reliably evaluated in this study, given the small sample size, but this is a common challenge for similar works in this population. The effect of potentially therapeutic drugs, such as SSRIs, on neuronal activation during affect recognition also remains unclear and could be clarified in future studies.

Additionally, it can be observed from our descriptive data that the TBI-I group may have sustained more severe TBI injuries than the TBI-N group, although these group differences did not reach statistical significance. The majority of injury severity data was obtained via self or family report, which is often a limitation due to lack of access to medical records or weak documentation in records. It may be that individuals with more severe injuries are more likely to exhibit affect recognition deficits. Any differences in injury severity between the TBI-I and TBI$\mathrm{N}$ would therefore potentially confound or magnify group differences, independent of processes related to emotion recognition.

Finally, the analytical approach required for the neuroimaging task used in this study did not allow isolation of the emotion from the face. Activation responses to emotional and neutral faces were combined and subtracted from a scrambled face with a central fixation point. As a result, brain activation reflected both general and emotional face processing. Although activation in the right fusiform gyrus was associated with performance on the facial affect recognition tasks, we cannot differentiate whether brain activation in this region was specific to emotion processing, or general face processing, or both. In order to establish a better understanding of the brain mechanisms specific to facial expressions per se after TBI, future studies should employ tasks 
that allow direct comparisons between emotional and neutral faces. Additionally, future studies may also want to compare brain responses to different emotions, since various emotions have been shown to engage different regions of the brain (Fusar-Poli et al. 2009) . Studies that want to make comparisons across emotions should consider neuroimaging tasks that include all of 6 of the universal facial (happy, sad, angry, fearful, surprise and disgust) for a more detailed understanding of the mechanisms involved in processing specific emotions.

\section{CONCLUSION}

In summary, this is the first study to examine the neurobiological underpinnings of facial affect recognition deficits in the TBI population. These preliminary findings indicate decreased activity in the fusiform gyrus as an underlying mechanism, which may implicate deficits in holistic face processing. This finding should be replicated with a larger sample size and evaluated in conjunction with tasks that serve as behavioral markers for holistic face processing. Additionally, in light of previous findings that suggest patients with impaired facial affect recognition have compromised psychosocial adjustment and deficits with executive functioning, researchers may also want to consider examining associations of brain activation and holistic processing with these variables (Knox and Douglas 2009; Yim et al. 2013). Elucidating the neurobiological and behavioral mechanisms associated with facial affect recognition deficits after TBI will hopefully improve the future rehabilitation landscape for these impairments.

\section{Acknowledgements}

We would like to thank Dr. Ruben Gur and his colleagues for generously sharing their fMRI neuroimaging facial affect recognition task. This research was funded by the Indiana University Collaborative Research Grant fund of the Office of the Vice President for Research and by the Indiana University Signature Center: Brain Rehabilitation, Advanced Imaging, and Neuroscience.

\section{Conflicts of Interest}


All authors declare that they have no conflict of interest.

\section{Compliance with Ethical Standards}

All procedures followed were in accordance with the ethical standards of the Indiana University

Institutional Review Board and with the Helsinki Declaration of 1964, and the applicable

revisions at the time of the investigation. Informed consent was obtained from all participants for being included in the study.

\section{References}

Adolphs, R. (2002). Recognizing emotion from facial expressions: Psychological and neurological mechanisms. Behavioral and Cognitive Neuroscience Reviews, 1(1), 21.

Adolphs, R., Gosselin, F., Buchanan, T. W., Tranel, D., Schyns, P., \& Damasio, A. R. (2005). A mechanism for impaired fear recognition after amygdala damage. Nature, 433(7021), 6872.

Avidan, G., Tanzer, M., \& Behrmann, M. (2011). Impaired holistic processing in congenital prosopagnosia. Neuropsychologia, 49(9), 2541-2552.

Babbage, D. R., Yim, J., Zupan, B., Neumann, D., Tomita, M. R., \& Willer, B. (2011). Metaanalysis of facial affect recognition difficulties after traumatic brain injury. Neuropsychology, 25(3), 277.

Bornhofen, C., \& McDonald, S. (2008). Comparing strategies for treating emotion perception deficits in traumatic brain injury. The Journal of Head Trauma Rehabilitation, 23(2), 103115.

Bourke, C., Douglas, K., \& Porter, R. (2010). Processing of facial emotion expression in major depression: a review. Australian and New Zealand Journal of Psychiatry, 44(8), 681-696.

Busigny, T., Joubert, S., Felician, O., Ceccaldi, M., \& Rossion, B. (2010). Holistic perception of the individual face is specific and necessary: Evidence from an extensive case study of acquired prosopagnosia. Neuropsychologia, 48(14), 4057-4092.

Busigny, T., Van Belle, G., Jemel, B., Hosein, A., Joubert, S., \& Rossion, B. (2014). Facespecific impairment in holistic perception following focal lesion of the right anterior temporal lobe. Neuropsychologia, 56, 312-333.

Centers for Disease Control and Prevention. last accessed January 23, 2015. http://www.cdc.gov/TraumaticBrainInjury/index.html. 
Curran, C. A., Ponsford, J. L., \& Crowe, S. (2000). Coping strategies and emotional outcome following traumatic brain injury: A comparison with orthopedic patients. The Journal of Head Trauma Rehabilitation, 15(6), 1256-1274.

Dalton, K. M., Nacewicz, B. M., Johnstone, T., Schaefer, H. S., Gernsbacher, M. A., Goldsmith, H., et al. (2005). Gaze fixation and the neural circuitry of face processing in autism. Nature Neuroscience, 8(4), 519-526.

Dolan, R. J., Morris, J. S., \& de Gelder, B. (2001). Crossmodal binding of fear in voice and face. Proceedings of the National Academy of Sciences, 98(17), 10006-10010.

Edwards, J., Jackson, H. J., \& Pattison, P. E. (2002). Emotion recognition via facial expression and affective prosody in schizophrenia: a methodological review. Clinical Psychology Review, 22(6), 789-832.

Fann, J. R., Bombardier, C. H., Dikmen, S., Esselman, P., Warms, C. A., Pelzer, E., et al. (2005). Validity of the Patient Health Questionnaire-9 in assessing depression following traumatic brain injury. The Journal of Head Trauma Rehabilitation, 20(6), 501-511.

Fusar-Poli, P., Placentino, A., Carletti, F., Landi, P., Allen, P., Surguladze, S., et al. (2009). Functional atlas of emotional faces processing: a voxel-based meta-analysis of 105 functional magnetic resonance imaging studies. Journal of Psychiatry \& Neuroscience: JPN, 34(6), 418.

Ganel, T., Valyear, K. F., Goshen-Gottstein, Y., \& Goodale, M. A. (2005). The involvement of the "fusiform face area" in processing facial expression. Neuropsychologia, 43(11), 1645-1654.

Gur, R. C., Sara, R., Hagendoorn, M., Marom, O., Hughett, P., Macy, L., et al. (2002a). A method for obtaining 3-dimensional facial expressions and its standardization for use in neurocognitive studies Journal of Neuroscience Methods, 115(2), 137-143.

Gur, R. E., McGrath, C., Chan, R. M., Schroeder, L., Turner, T., Turetsky, B. I., et al. (2002b). An fMRI study of facial emotion processing in patients with schizophrenia. The American Journal of Psychiatry, 159(12), 1992-1999.

Harms, M. B., Martin, A., \& Wallace, G. L. (2010). Facial emotion recognition in autism spectrum disorders: a review of behavioral and neuroimaging studies. Neuropsychology Review, 20(3), 290-322.

Kanwisher, N., \& Yovel, G. (2006). The fusiform face area: a cortical region specialized for the perception of faces. Philosophical Transactions of the Royal Society B: Biological Sciences, 361(1476), 2109-2128.

Klin, A., Jones, W., Schultz, R., Volkmar, F., \& Cohen, D. (2002). Visual fixation patterns during viewing of naturalistic social situations as predictors of social competence in individuals with autism. Archives of General Psychiatry, 59(9), 809-816.

Knox, L. a., \& Douglas, J. (2009). Long-term ability to interpret facial expression after traumatic brain injuryand its relation to social integration. Brain and Cognition, 69, 442-449.

Kroenke, K., Spitzer, R. L., \& Williams, J. B. W. (2001). The PHQ-9. Journal of General Internal Medicine, 16(9), 606-613.

Li, H., Chan, R. C., McAlonan, G. M., \& Gong, Q.-y. (2010). Facial emotion processing in schizophrenia: a meta-analysis of functional neuroimaging data. Schizophrenia Bulletin, 36(5), 1029-1039.

Marsh, N., Kersel, D., Havill, J., Sleigh, J. (1998). Caregiver burden at 6 months following severe traumatic brain injury. Brain Injury, 12(3), 225-238.

Maurer, D., Le Grand, R., \& Mondloch, C. J. (2002). The many faces of configural processing. Trends in Cognitive Sciences, 6(6), 255-260.

McIntire, K. A., Danforth, M. M., \& Schneider, H. G. (1997). Measuring cue perception: Assessment of reliability and validity. Paper presented at the Meeting of the Southeastern Psychological Association, Atlanta, GA, 
Narumoto, J., Okada, T., Sadato, N., Fukui, K., \& Yonekura, Y. (2001). Attention to emotion modulates fMRI activity in human right superior temporal sulcus. Cognitive Brain Research, 12(2), 225-231.

Neumann, D., Babbage, D. R., Zupan, B., \& Willer, B. (2015). A randomized controlled trial of emotion recognition training after traumatic brain injury. The Journal of Head Trauma Rehabilitation, 30(3), E12-23.

Neumann, D., Keiski, M. A., McDonald, B. C., \& Wang, Y. (2014a). Neuroimaging and facial affect processing: implications for traumatic brain injury. Brain Imaging and Behavior, 8(3), 460-473.

Neumann, D., Zupan, B., Babbage, D. R., Radnovich, A. J., Tomita, M., Hammond, F., et al. (2012). Affect recognition, empathy, and dysosmia after traumatic brain injury. Archives of Physical Medicine and Rehabilitation, 93(8), 1414-1420.

Neumann, D., Zupan, B., Malec, J. F., \& Hammond, F. (2014b). Relationships between alexithymia, affect recognition, and empathy after traumatic brain injury. The Journal of Head Trauma Rehabilitation, 29(1), E18-27.

Nowicki, S., \& Duke, M. P. (1994). Individual differences in the nonverbal communication of affect: The Diagnostic Analysis of Nonverbal Accuracy Scale. Journal of Nonverbal Behavior, 18(1), 9-35.

Nowicki, S., \& Mitchell, J. (1998). Accuracy in identifying affect in child and adult faces and voices and social competence in preschool children. Genetic, Social, and General Psychology Monographs, 124(1), 39-59.

Ogai, M., Matsumoto, H., Suzuki, K., Ozawa, F., Fukuda, R., Uchiyama, I., et al. (2003). fMRI study of recognition of facial expressions in high-functioning autistic patients. Neuroreport, 14(4), 559-563.

Pelphrey, K. A., Sasson, N. J., Reznick, J. S., Paul, G., Goldman, B. D., \& Piven, J. (2002). Visual scanning of faces in autism. Journal of Autism and Developmental Disorders, 32(4), 249-261.

Phillips, M. L., Drevets, W. C., Rauch, S. L., \& Lane, R. (2003). Neurobiology of emotion perception I: The neural basis of normal emotion perception. Biological Psychiatry, 54(5), 504-514.

Piggot, J., Kwon, H., Mobbs, D., Blasey, C., Lotspeich, L., Menon, V., et al. (2004). Emotional attribution in high-functioning individuals with autistic spectrum disorder: a functional imaging study. Journal of the American Academy of Child and Adolescent Psychiatry, 43(4), 473-480.

Rossion, B. (2013). The composite face illusion: A whole window into our understanding of holistic face perception. Visual Cognition, 21(2), 139-253.

Rossion, B., \& Gauthier, I. (2002). How does the brain process upright and inverted faces? Behavioral and Cognitive Neuroscience Reviews, 1(1), 63-75.

Sabatinelli, D., Fortune, E. E., Li, Q., Siddiqui, A., Krafft, C., Oliver, W. T., et al. (2011). Emotional perception: Meta-analyses of face and natural scene processing. NeuroImage, 54(3), 2524-2533.

Schiltz, C., Dricot, L., Goebel, R., \& Rossion, B. (2010). Holistic perception of individual faces in the right middle fusiform gyrus as evidenced by the composite face illusion. Journal of Vision, 10(2), 25.

Spell, L. A., \& Frank, E. (2000). Recognition of nonverbal communication of affect following traumatic brain injury. Journal of Nonverbal Behavior, 24(4), 285-300.

Spielberger, C. D., Gorsuch, R. L., and , \& R.E., L. (1970). Manual for the State-Trait Anxiety Inventory. Palo Alto, CA: Consulting Psychologists Press.

Spielberger, C. D., Reheiser, E. C., Ritterband, L. M., Sydeman, S. J., \& Unger, K. K. (1995). Assessment of Emotional States and Personality Traits: Measuring Psychological Vital 
Signs, Clinical Personality Assessment: Practical Approaches. In Butcher, J.N. (Ed.): New York: Oxford University Press.

Spikman, J. M., Milders, M. V., Visser-Keizer, A. C., Westerhof-Evers, H. J., Herben-Dekker, M., \& van der Naalt, J. (2013). Deficits in facial emotion recognition indicate behavioral changes and impaired self-awareness after moderate to severe traumatic brain injury. PloS one, 8(6), e65581.

Vassallo, S., Cooper, S. L., \& Douglas, J. M. (2009). Visual scanning in the recognition of facial affect: Is there an observer sex difference? Journal of Vision, 9(3), 11.

Wang, A. T., Dapretto, M., Hariri, A. R., Sigman, M., \& Bookheimer, S. Y. (2004). Neural correlates of facial affect processing in children and adolescents with autism spectrum disorder. Journal of the American Academy of Child and Adolescent Psychiatry, 43(4), 481-490.

Winston, J. S., Henson, R., Fine-Goulden, M. R., \& Dolan, R. J. (2004). fMRI-adaptation reveals dissociable neural representations of identity and expression in face perception. Journal of Neurophysiology, 92(3), 1830-1839.

Yim, J., Babbage, D. R., Zupan, B., Neumann, D., \& Willer, B. (2013). The relationship between facial affect recognition and cognitive functioning after traumatic brain injury. Brain Injury, 27(10), 1155-1161.

Table 1. Sample characteristics and group comparisons. LOC=Loss of Consciousness;

PTA=posttraumatic amnesia; PHQ-9=Patient Health Questionnaire (measure of depression);

STAI=State Trait Anxiety Inventory. STAI-State norms: Male=35.72 (10.40); Female=35.20

(10.02). STAI-Trait norms: Male=34.89 (9.19); Female=34.79 (9.22).

\begin{tabular}{|l|c|c|c|c|}
\hline & $\begin{array}{c}\text { TBI -I } \\
\text { Mean (s.d.) } \\
\text { or \% }\end{array}$ & $\begin{array}{c}\text { TBI- N } \\
\text { Mean (s.d.) } \\
\text { or \% }\end{array}$ & $\begin{array}{c}\text { HC } \\
\text { Mean (s.d.) } \\
\text { or \% }\end{array}$ & Difference \\
\hline Age & 37 & 43.58 & 38.25 & $\mathrm{~F}=0.833$, \\
& $(11.77)$ & $(13.46)$ & $(13.9)$ & $\mathrm{p}=0.44$ \\
\hline
\end{tabular}




\begin{tabular}{|c|c|c|c|c|}
\hline Sex (males) & $54.5 \%$ & $58 \%$ & $50 \%$ & $\begin{aligned} \chi^{2} & =0.188, \\
p & =0.919\end{aligned}$ \\
\hline Education (years) & $\begin{array}{l}15.73 \\
(2.72)\end{array}$ & $\begin{array}{l}14.92 \\
(1.78)\end{array}$ & $\begin{array}{c}15.5 \\
(2.43)\end{array}$ & $\begin{array}{c}F=0.374 \\
p=0.69\end{array}$ \\
\hline Years Post-Injury & $\begin{array}{c}8.88 \\
(8.70)\end{array}$ & $\begin{array}{l}12.67 \\
(9.71)\end{array}$ & N/A & $\begin{array}{c}t=-0.964 \\
p=0.35\end{array}$ \\
\hline $\begin{array}{l}\text { LOC (days) } \\
(n=19)\end{array}$ & $\begin{array}{l}22.20 \\
(0-90)\end{array}$ & $\begin{array}{c}12.90 \\
(.08-42)\end{array}$ & N/A & $\begin{array}{l}\mathrm{t}=0.870 \\
\mathrm{p}=0.40\end{array}$ \\
\hline $\begin{array}{l}\text { PTA (days) } \\
(\mathrm{n}=15)\end{array}$ & $\begin{array}{c}32.83 \\
(14-60)\end{array}$ & $\begin{array}{l}16.89 \\
(2-42)\end{array}$ & N/A & $\begin{array}{l}\mathrm{t}=2.08 \\
\mathrm{p}=0.06\end{array}$ \\
\hline $\begin{array}{rr}\text { PHQ-9 } & \text { Minimal } \\
\text { Mild } \\
\text { Moderate } \\
\text { Moderate-Severe } \\
\text { Severe }\end{array}$ & $\begin{array}{c}7.27(7.19) \\
45.5 \% \\
18.2 \% \\
18.2 \% \\
18.2 \% \\
0 \%\end{array}$ & $\begin{array}{c}5.83(5.89) \\
50 \% \\
25 \% \\
16.7 \% \\
0 \% \\
8.3 \%\end{array}$ & $\begin{array}{c}1.58(5.79) \\
91.7 \% \\
8.3 \% \\
0 \% \\
0 \% \\
0 \%\end{array}$ & $\begin{array}{l}\chi^{2}=5.00 \\
(p=.082)\end{array}$ \\
\hline $\begin{array}{r}\text { STAI-State Anxiety } \\
\text { Mean Rank } \\
\text { STAI-Trait Anxiety } \\
\text { Mean Rank }\end{array}$ & $\begin{array}{c}35.73(16.03) \\
19.05 \\
38.73(15.33) \\
19.00 \\
\end{array}$ & $\begin{array}{c}33.17(14.52) \\
18.46 \\
38.17(14.99) \\
18.63 \\
\end{array}$ & $\begin{array}{c}27.42(6.44) \\
16.58 \\
33.50(7.9) \\
16.46 \\
\end{array}$ & $\begin{array}{l}\chi^{2}=.373 \\
(p=.830) \\
\chi^{2}=.423 \\
(p=.810\end{array}$ \\
\hline
\end{tabular}

Table 2. Performance on Facial Recognition Tasks. Because the DANVA and Neuroimaging task accuracy variances were unequal across the groups, means, medians, and percentiles are presented for these variables for the entire sample, as are the mean, standard deviation, variance, and mean ranks for each group.

\begin{tabular}{|l|c|c|c|c|}
\hline $\begin{array}{l}\text { Facial Affect Recognition } \\
\text { test }\end{array}$ & $\begin{array}{c}\text { All subjects } \\
\text { Mean (s.d.) } \\
\text { Median } \\
\end{array}$ & $\begin{array}{c}\text { TBI-I } \\
\text { Mean (s.d.) } \\
\text { Variance } \\
\text { 25 }\end{array}$ & $\begin{array}{c}\text { TBI-N } \\
\text { Mean (s.d.) } \\
\text { Variance } \\
\text { Mean }\end{array}$ & $\begin{array}{c}\text { HC } \\
\text { Mean (s.d.) } \\
\text { Variance } \\
\text { Mean Rank }\end{array}$ \\
& $75^{\text {th }}$ percentile & & & \\
\hline DANVA2-AF (z scores) & $-0.72(1.24)$ & $-2.18(1.07)$ & $-0.07(0.64)$ & $-0.04(0.47)$ \\
& -0.26 & 1.14 & 0.409 & 0.220 \\
& -1.22 & 6.00 & 22.88 & 24.13 \\
\hline
\end{tabular}




\begin{tabular}{|l|c|c|c|c|}
\hline & 0.17 & & & \\
\hline Neuroimaging task & $87(6.9)$ & $82.94(6.6)$ & $86.30(8.2)$ & $91.63(2.14)$ \\
accuracy (\%) & 90 & 42.99 & 64.48 & 4.57 \\
& 83 & 11.50 & 16.29 & 25.67 \\
\hline $\begin{array}{l}\text { Neuroimaging task } \\
\text { reaction time (seconds; } \\
\text { weighted calculation based } \\
\text { on \% of target and non- }\end{array}$ & $.96(.20)$ & $1.07(.18)$ & $1.00(.22)$ & $.83(.12)$ \\
target stimuli ) & & & & \\
\hline
\end{tabular}

Table 3. Statistically Significant fMRI Clusters (values are for peak voxel within cluster)

\begin{tabular}{|c|c|c|c|c|c|c|c|c|}
\hline \multicolumn{5}{|c|}{ Cluster-level } & \multicolumn{4}{|c|}{ Peak-level } \\
\hline Cluster & $\begin{array}{l}\text { PFWE- } \\
\text { corr }\end{array}$ & $\begin{array}{l}\text { QFDR- } \\
\text { corr }\end{array}$ & $\mathrm{k}_{\mathrm{E}}$ & Puncorr & $\mathrm{T}$ & $\begin{array}{c}\text { Peak MNI } \\
\text { coordinates } \\
(\mathrm{x} \mathrm{y} \mathrm{z})\end{array}$ & $\begin{array}{l}\text { Region description } \\
\text { (for cluster peak) }\end{array}$ & $\begin{array}{c}\text { Brodmann } \\
\text { Area } \\
\text { (BA) }\end{array}$ \\
\hline \multicolumn{9}{|c|}{ Main Effect for Facial Affect Recognition across All Groups Overall $\mathrm{p}_{\text {crit }}<0.001$, Cluster-level $\mathrm{p}_{\text {uncorr }}<0.05$} \\
\hline 1 & $<0.001$ & $<0.001$ & 10432 & $<0.001$ & 17.00 & $(38,-46,-20)$ & Right Fusiform Gyrus & 37 \\
\hline 2 & $<0.001$ & $<0.001$ & 4956 & $<0.001$ & 14.05 & $(48,14,28)$ & Right Inferior Frontal Gyrus & 9 \\
\hline
\end{tabular}




\begin{tabular}{ccccccccc}
3 & $<0.001$ & $<0.001$ & 2536 & $<0.001$ & 11.55 & $(-6,12,52)$ & Left Superior Frontal Gyrus & 8 \\
4 & $<0.001$ & $<0.001$ & 5913 & $<0.001$ & 9.63 & $(-40,6,32)$ & Left Inferior Frontal Gyrus & 9 \\
5 & $<0.001$ & $<0.001$ & 1399 & $<0.001$ & 6.29 & $(-6,-26,-8)$ & L Midbrain & N/A \\
6 & 0.202 & 0.048 & 88 & 0.018 & 6.03 & $(2,-54,-34)$ & Right Cerebellum & N/A \\
7 & 0.002 & $<0.001$ & 296 & $<0.001$ & 5.62 & $(18,4,12)$ & Right Putamen & N/A \\
8 & 0.154 & 0.040 & 98 & 0.013 & 4.46 & $(30,-18,-2)$ & Right Putamen & N/A \\
9 & 0.150 & 0.040 & 99 & 0.013 & 4.39 & $(-50,-48,10)$ & Left Superior Temporal Gyrus & 22 \\
10 & 0.264 & 0.059 & 78 & 0.025 & 4.36 & $(22,-4,-12)$ & Right Amygdala & N/A \\
\hline HC $>$ TBI-I Overall pcrit $<0.001$, Cluster-level puncorr $<0.05$ & & & 37 \\
\hline
\end{tabular}

Figure 1. Neuroimaging task. This figure is a representation of images presented during the fearful condition. 


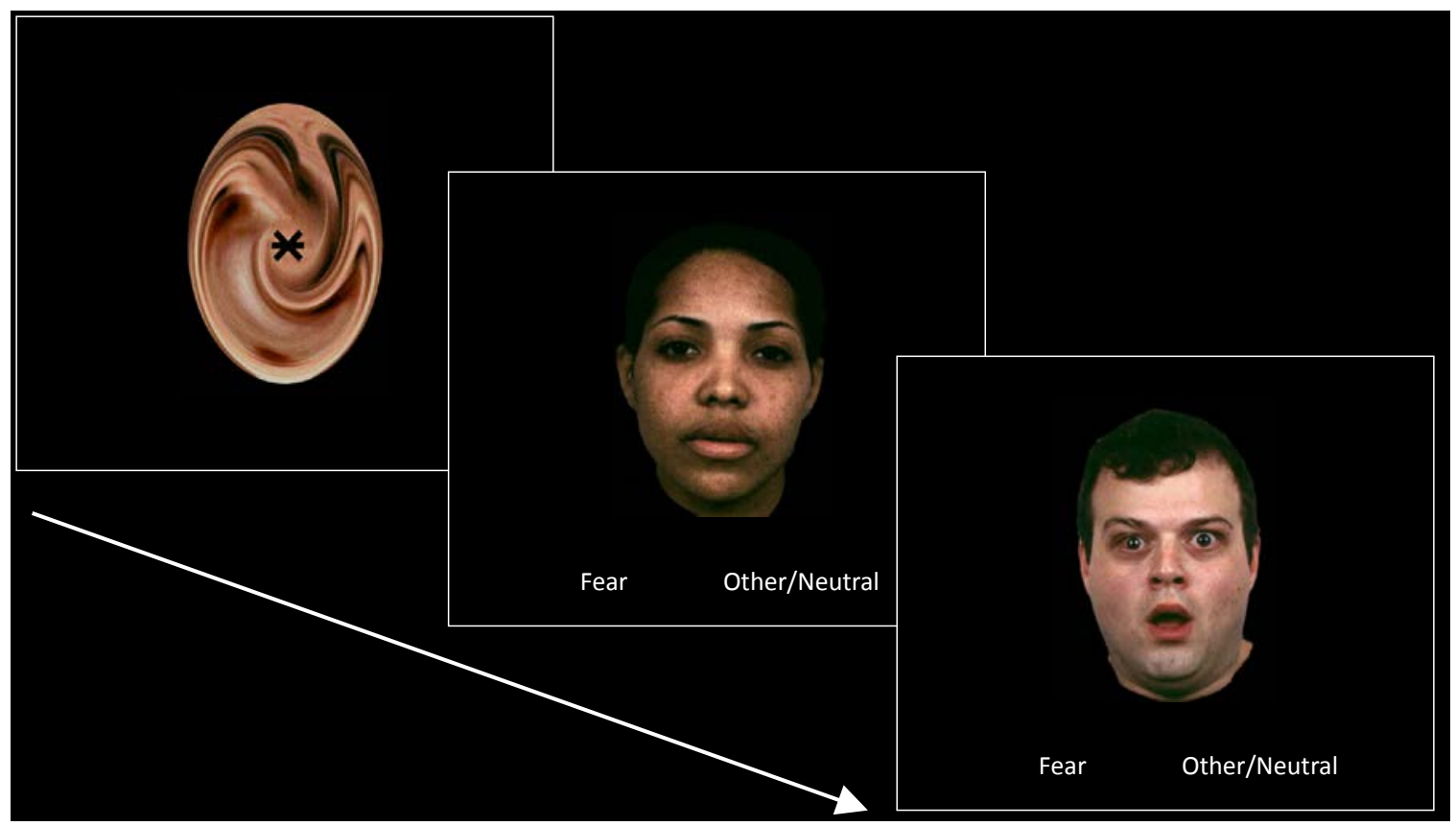

Figure 2. Main Effect of fMRI Facial Affect Recognition across All Groups.

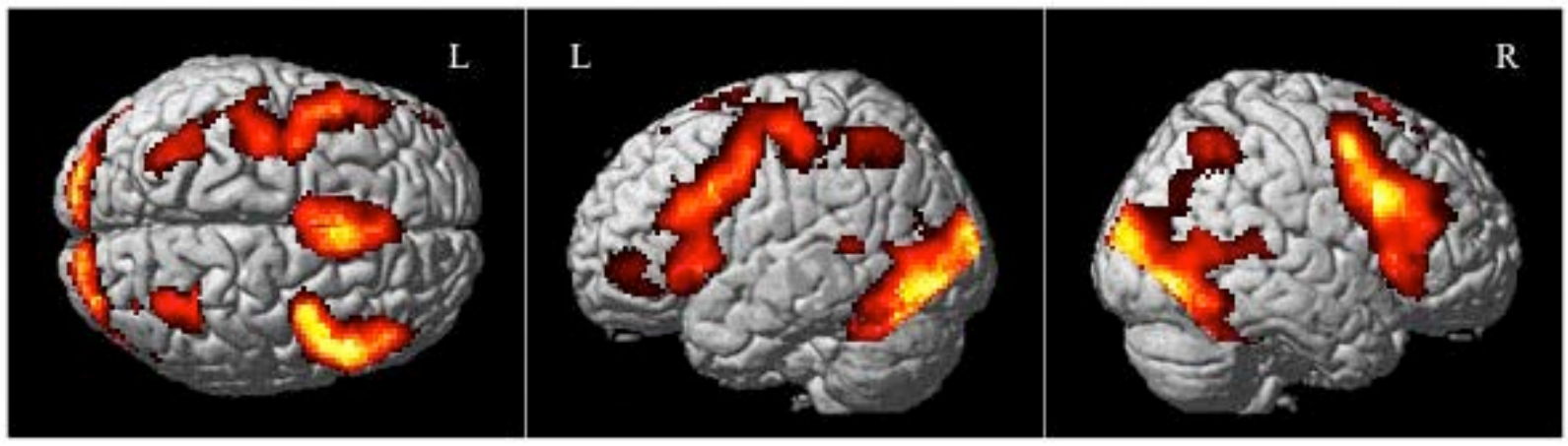


Figure 3. Greater Right Fusiform Activation in HC Relative to TBI-I
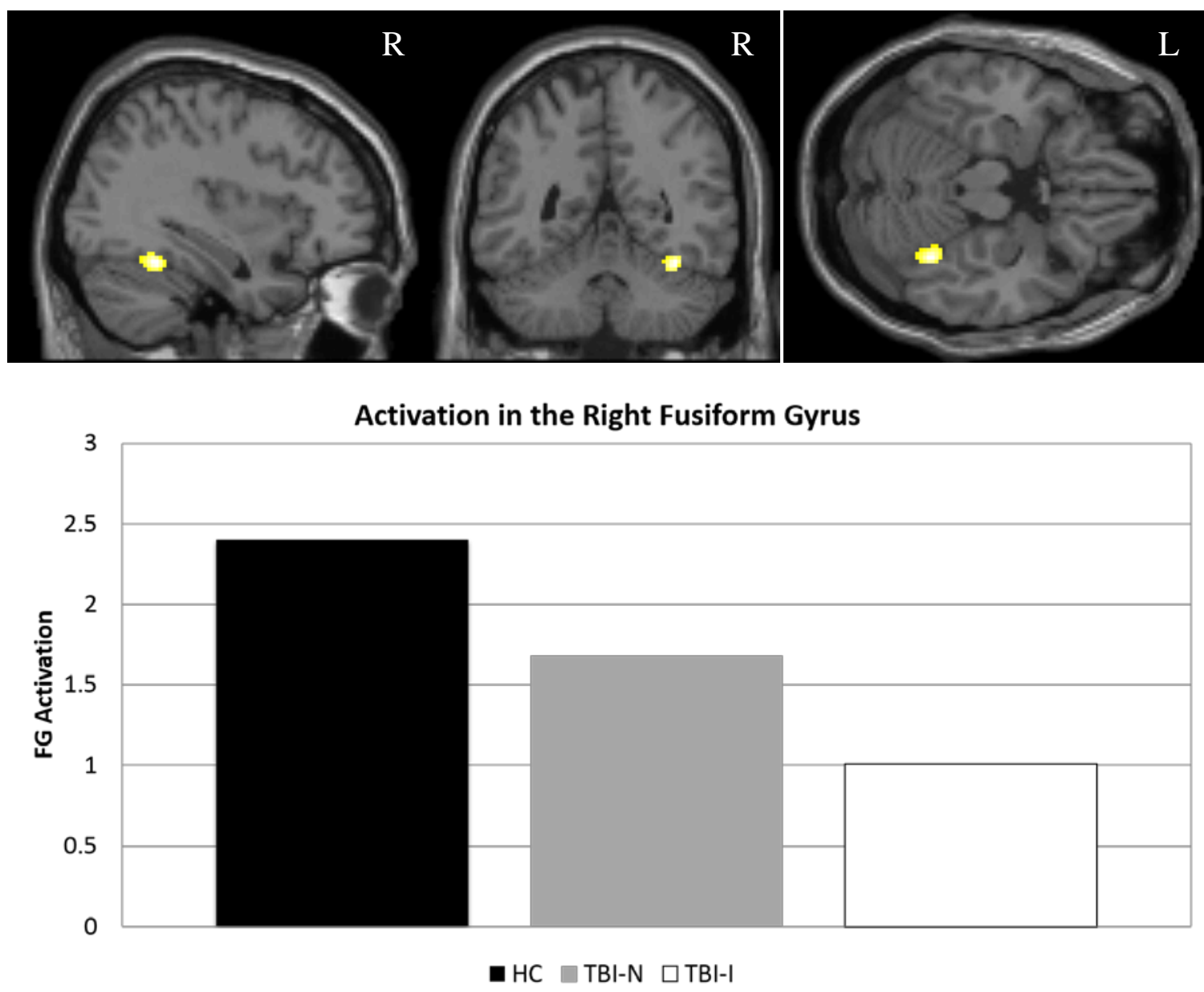